\title{
UVA-induced protection of skin through the induction of heme oxygenase-1
}

\author{
Yuancai Xiang ${ }^{1}$, Gang Liư ${ }^{2}$ Li Yang ${ }^{1}$ Julia Li Zhong ${ }^{1,2, *}$ \\ ${ }^{1}$ The "111 Project" Laboratory of Biomechanics \& Tissue Repair Engineering, Key Laboratory of Biorheological Science and \\ Technology, Ministry of Education, College of Bioengineering, Chongqing University, Chongqing, China; \\ ${ }^{2}$ Dermatology Institute, Sichuan Provincial People's Hospital, Chengdu, China.
}

\begin{abstract}
Summary UVA (320-400 $\mathrm{nm})$ and UVB $(290-320 \mathrm{~nm})$ are the major components of solar UV irradiation, which is associated with various pathological conditions. UVB causes direct damage to DNA of epidermal cells and is mainly responsible for erythema, immunosuppression, photoaging, and skin cancer. UVA has oxidizing properties that can cause damage or enhance UVB damaging effects on skin. On the other hand, UVA can also lead to high levels of heme oxygenase-1 (HO-1) expression of cells that can provide an antioxidant effect on skin as well as anti-inflammatory properties in mammals and rodents. Therefore, this review focuses on the potential protection of UVA wavebands for the skin immune response, instead of mechanisms that underlie UVA-induced damage. Also, the role of HO-1 in UVA-mediated protection against UVB-induced immunosuppression in skin will be summarized. Thus, this review facilitates further understanding of potential beneficial mechanisms of UVA irradiation, and using the longer UVA (UVA1, 340-400 nm) in combination with $\mathrm{HO}-1$ for phototherapy and skin protection against sunlight exposure.
\end{abstract}

Keywords: UVA, heme oxygenase-1, immunoprotection

\section{Introduction}

The solar ultraviolet (UV) is a spectrum of sunlight that causes photoaging, DNA damage, gene mutation, and even skin cancer (1). UV light that reaches the earth is divided into ultraviolet A (UVA, 320-400 nm) and ultraviolet B (UVB, 290-320 nm), and the UVA waveband can be further divided into UVA1 (340-400 nm) and UVA2 (320-340 nm) (2,3). UVB constitutes less than $10 \%$ of solar UV, and it is generally thought to be the most deleterious radiation of sunlight and the major wavelength involved in erythema, immunosuppression and carcinogenesis outcomes (4). UVA constitutes over $90 \%$ of solar UV. It can penetrate

\footnotetext{
*Address correspondence to:

Dr. Julia Li Zhong, The "111 Project" Laboratory of Biomechanics \& Tissue Repair Engineering, Key Laboratory of Biorheological Science and Technology, Ministry of Education, College of Bioengineering, Chongqing University, Chongqing 400044, China. e-mail: jlzhong@cqu.edu.cn
}

deep into skin, generate reactive oxygen species (ROS) through reacting with cellular chromophores, initiate photochemical and photobiological events (such as oxidation of various molecules and DNA damage), and consequently cause immunosuppression $(1,2,5)$. It was found that UVA could cause immunosuppression in human skin, and sunscreen studies also indicated that a broad-spectrum sunscreen (blocking both UVA and UVB) provides better protection compared to a narrow blocker (only UVB), thus showing an important role of UVA in inducing immunosuppression (6-8). However, recently, a growing number of studies indicated that UVA radiation could provide immunoprotection and also inhibit UVB-induced immunosuppression through modulation of various cutaneous cytokines and enzymes, such as heme oxygenase-1 (HO-1) $(2,9,10)$.

$\mathrm{HO}-1$ is a potent anti-inflammatory intracellular mediator, which catalyzes the degradation of heme to iron, biliverdin, and carbon monoxide (CO) (11). High expression of HO-1 is observed in many types of cells after stimulation by UVA radiation, nitric oxide, heavy metals, and many other oxidants $(1,2)$. Induction of HO-1 causes anti-inflammatory and anti-apoptotic 
properties. Especially, the anti-inflammatory capacity of HO-1 can exert immunomodulation of cell responses and alleviate a number of chronic inflammatory diseases (12). Tyrrell and Reeve suggested that the protection of skin by UVA irradiation with appropriate doses and wavelengths may be linked to UVA-induced HO-1 $(2,13)$. In recent years, studies of the mechanism of UVA induced HO-1 immunoprotection have made great progress, for example, IL- 6 and estrogen receptor- $\beta$ (Er- $\beta$ ) are involved in mediating immunoprotection by UVA radiation, and gender difference is one of the factors that influence UVA-induced immunoprotection $(9,14,15)$. So it is necessary to summarize recent developments of studies of UVA induced HO-1 expression and its protective effects on skin. In this article, HO-1 immunoprotection in skin, and its relationship with UVA induced immunoprotection will be elaborated, and UVA phototherapy will also be briefly mentioned due to its beneficial role in treating some skin diseases.

\section{The relationship between UVA waveband, dose and immune function}

There is a great deal of disagreement on UVA-induced immune function, which could be attributed to UVA waveband and dose. The wide spectral waveband of UVA (320-400 nm) could react with different chromophores in skin, leading to different effects on the immune system of skin (16). It was found that pretreatment with UVA1 (340-400 nm) may offer partial protection against UVB-induced decrease of immunization reaction to epidermis allergens (17). In another study, the result indicated that UVA1 did not elicit immunosuppression, however, UVA did induce immunosuppression that might be caused by UVA2 (shorter wavelength of UVA, 320-340 nm) (18). UVA1 and UVA2 might show differences in the regulation of immune functions: UVA1 may provide protection in the immune system; UVA2 is related to UVB in some parts of the immune response, such as immunosuppression, since their wavebands are adjacent $(2,18,19)$.

In addition to the wavelength, UVA irradiation with different dosages may cause various biological effects on the immune system. Halliday et al. found that irradiation with a low dose of UVA $(8.4 \mathrm{~kJ} /$ $\mathrm{m}^{2}$ ) on humans could enhance immune memory; a medium dose of UVA $\left(16.8 \mathrm{~kJ} / \mathrm{m}^{2}\right)$ might have immunosuppressive activity, but higher doses of UVA (i.e., $33.6 \mathrm{~kJ} / \mathrm{m}^{2}$ ) could protect skin from UVB damage (20). Similar results have been obtained using mice to show that UVA acts as an immunosuppressor at a lower dose $\left(18 \mathrm{~kJ} / \mathrm{m}^{2}\right)$ and an immunoprotector at a higher dose $\left(34 \mathrm{~kJ} / \mathrm{m}^{2}\right)$ (21). An in vivo study, using hairless mice, identified UVA doses, between 16 and $580 \mathrm{~kJ} /$ $\mathrm{m}^{2}$, could provide immunoprotection against UVBinduced immunosuppression. However, UVA might be immunosuppressive at doses over $600 \mathrm{~kJ} / \mathrm{m}^{2}$ (22). We suggest that the discrepancy of immuno-functions among those studies may be due to the non-uniformity of the UV radiometer used for dose measurement and the definition of dose levels (low, medium and high). The different mice strain may also account for the discrepancy as well.

Recently, our study indicated that the increase of HO-1 levels by interference of Bach1 (BTB and CNC homology 1) might protect human skin keratinocytes from damage by a high dose of UVA (400-500 kJ/m²) irradiation, while this increment had no effect on the protection for low to medium doses (100 and 250 $\left.\mathrm{kJ} / \mathrm{m}^{2}\right)(23,24)$. Nevertheless, in vivo studies of UVA immune responses mostly adopted mice models, and the immune responses of human skin are currently unclear due to too few studies.

\section{UVA induced HO-1 protection in skin cells}

UVA radiation alters a series of antioxidant pathways in skin cells, and one of the robust pathways is through the induction of HO-1 expression. HO-1 plays a vital role in protecting various cells and tissues against oxidative stress by virtue of its anti-inflammatory, anti-apoptotic, and anti-proliferative features (2). Also, it is a powerful immunomodulator which could eliminate some inflammatory responses (25). HO-1 can be induced not only by UVA radiation but also by many other stimuli, including heavy metals, endotoxin, $\mathrm{H}_{2} \mathrm{O}_{2}$, heat shock, LPS, inflammatory cytokines, and other antioxidants $(1,2,26)$; The level of HO-1 expression depends on the type of cells. In this section, we will introduce the functions of HO-1 in skin and its role in UVA-induced immunoprotection.

\subsection{HO-1 and its functions in skin}

HO-1 is a rate-limiting enzyme in heme metabolism and can cleave heme into three products: biliverdin, $\mathrm{CO}$, and ferrous iron. Biliverdin is further reduced by biliverdin reductase to bilirubin. Biliverdin and bilirubin are recognized as potent antioxidants by scavenging ROS (27). CO, as biliverdin, contributes substantially to the anti-inflammatory properties of HO-1 by suppressing pro-inflammatory cytokines and also has tolerogenic actions in adaptive immune responses $(11,13)$. Ferrous iron is released during the breakdown of free heme by HO-1, but this molecule is rapidly removed by ferritin. It is suggested that increased ferritin expression in conjunction with HO-1 expression may contribute to the additional protection by HO-1 (28-30).

The activation of HO-1 has been recognized as a sensitive marker for oxidative stress to cells, and it is involved in an adaptive protective response against oxidative damage, such as UV irradiation $(1,2)$. Recently, many studies showed that HO-1 might 
provide protection through its upstream activation genes, such as transcription enhancer Nuclear factorerythroid-2-related factor 2 (Nrf2). For example, Hirota found that Nrf2 might play an important role in the protection of the skin against UVA irradiation in mice fibroblasts (31). Later, Nrf2-driven HO-1 expression was found to protect mouse skin cells from oxidative carcinogenesis (32). Our studies also showed that Nrf2 might protect human skin fibroblasts and keratinocytes from UVA irradiation $(23,33)$. These studies indicated that Nrf2-driven HO-1 expression may play a role in cellular protection. So far, these studies focused on the upstream regulation of HO-1. However, the protection provided by HO-1 through its downstream genes is yet to be identified.

\subsection{HO-1 in UVA-induced immunoprotection}

The induction of HO-1 may play an important role in the limiting inflammation and immune activation. For example, cutaneous HO-1 expression might represent a potent therapeutic approach for the treatment of $\mathrm{T}$ cell-dependent inflammatory dermatoses (34). It is also shown that HO-1 has immunomodulatory capacity in adaptive responses, and deficiency of HO-1 leads to development of chronic inflammatory pathology and widespread oxidative tissue injury (35). Lately, a study indicated that HO-1 can reduce the cutaneous Arthus reaction, an immune complexes-mediated disease that can result in edema, hemorrhage and neutrophil recruitment in the skin (36) (Table 1).

Using mice, Reeve VE et al. found that a suberythemogenic dose of UVA exposure could protect skin from the immunosuppressive effect of either UVB radiation or cis-urocanic acid (UCA). They indicated that the mechanism of UVA immunoprotection involves the induction of cutaneous HO-1 $(37,38)$. Moreover, they reported that UVA irradiation upregulated HO-1 in the dermis and epidermis of hairless mouse skin, and UVA-induced HO enzyme activity is protective against UVB-induced immunosuppression $(10,39)$. Further, they demonstrated that, with a constant UVB dose, UVA enrichment showed to be dose dependent for immunoprotection $(10,40)$. In their studies, through an HO inhibitor (tin protoporphyrin-IX), it was confirmed that $\mathrm{HO}$ is responsible for UVA-induced protection
$(10,40)$. Also, the refractoriness of UVA-induced protection from photoimmunosuppression was found to be correlated with HO-1 levels with repeated UVA exposure in Skh:HR-2 mice (41). The refractoriness of HO-1 was originally found in human skin fibroblast FEK4 cells following retreatment with a second dose of UVA radiation (42). Studies from our lab indicate that refractoriness of HO-1 might be linked to UVA-induced Nrf2 refractoriness in human skin fibroblast FEK4 cells (Zhong, unpublished data).

The immunoprotection of HO-1 is closely related to certain cytokines. The immunoprotection of UVAinduced HO-1 was found to be absent in IFN- $\gamma$ knockout mouse, indicating that IFN- $\gamma$ is involved in HO-1 induction (43). They also indicated that HO-1 could provide protection through its enzymatic product, CO. Utilizing a CO-releasing molecule to deliver $\mathrm{CO}$ to the skin, they found that $\mathrm{CO}$ concentration-dependently protected mice against the immunosuppression caused by solar simulated UV radiation and cis-UCA (44). Further studies indicated that UVA immunoprotection was linked to skin cyclic guanosine monophosphate (cGMP), which is activated by CO. The release of $\mathrm{CO}$ is due to HO-1 activation, and this suggests that HO-1 plays a role in UVA-induced immunoprotection (22). Moreover, the relationship between HO-1 inducibility and estrogen receptor- $\beta$ (Er- $\beta$ ) signaling was investigated. They found that the HO-1 gene was unresponsive to UVA induction in Er- $\beta-/-$ mice, and HO-1 inducibility and Er- $\beta$ signaling are interdependent requisite responses to the UVA waveband for its immunoprotection (9). Recently, they showed that IL-6 has an important photoimmunoprotective function through interaction with the HO-1 pathway, determining the immunologically advantageous actions of UVA radiation (14). These studies indicated the relevant pathway of HO-1 in the UVA-induced immune response, and will provide a guideline for more in depth understanding of the mechanism of HO-1 in UVAinduced immunoprotection.

\section{UVA Phototherapy}

Phototherapy using UVA has been successfully applied to the treatment of some cutaneous diseases, such as psoriasis, vitiligo and T-cell lymphoma $(45,46)$. UVA1

Table 1. Evidence of HO-1 in UVA-induced immunoprotection

\begin{tabular}{lll}
\hline Experimental subjects & Methods & Involved factors \\
\hline SHR1 and C57BL/6 & CHS & HO-1 and Er- $\beta$ \\
C57BL/6 & CHS & IL-6 and HO-1. \\
SHR1 & CHS & cGMP \\
SHR1 & CHS & UVA-induced HO activity \\
SHR1, C57BL/6, and IFN- $\gamma-/-$ & -- & IFN- $\gamma$ and HO-1 \\
SHR1 & CHS & CO released by HO activity
\end{tabular}

SHR: Skh:HR hairless mice; C57BL/6: C57BL/6 mice; IFN- $\gamma$-/-: IFN- $\gamma$-/- mice; CHS: Contact hypersensitivity; MED: Minimal erythema doses. 
has been shown to be more effective due to its deeper penetration into skin and less side effects, such as erythema, immunosuppression and carcinogenesis than UVA2. UVA1 can induce apoptosis of skin-infiltrating T cells, lead to T-cell depletion, and induce collagenase-1 expression in human dermal fibroblasts (47). These are thought to underlie the UVA1 therapeutics in several diseases, such as atopic dermatitis, inflammatory morphea and scleroderma (46). Several studies have also shown the effect of UVA1 on cytokine production. It suppresses proinflammatory cytokines such as TNF- $\alpha$ and IL-12 (46). It was also found that UVA1 can exert its beneficial effects in treating systemic lupus erythematosus (SLE) though decreasing IFN- $\gamma$, which has a pathogenic role in the development of SLE (48). Since UVA can provide immunoprotection via induction of HO-1 (long wave UVA is more effective in inducing HO-1 expression when compared with short wave UVA, Zhong, unpublished), we suggest that it would be more effective in treating skin diseases combining HO-1 with UVA1 in phototherapy. Obviously, prior to using HO-1 for the enhancement of UVA phototherapy, mechanisms of immunoprotection linked to HO-1 induction need to be further investigated, as suggested in the literature $(2,45,46)$. With the development of technology, using a narrow wavelength range or a mono wavelength of UVA phototherapy will become an efficient method in the treatment of localized and systemic skin disorders (49).

\section{Conclusions}

UVA irradiation may cause both beneficial (immunoprotection) and damaging (immunosuppression) effects on skin, which depend on wavelength, exposure dose, and UVA sources. In this review, the potential protection of specific UVA wavebands and doses to the skin immune response was briefly summarized. Since immunoprotection might be associated with longer wavelengths of UVA (340-400 nm), using narrow bands of UVA or a mono-wavelength in phototherapy can become more effective and have less side effects than using broad bands of UVA. Also, UVA induces high expression of anti-inflammatory enzyme HO-1, which provides beneficial effects on the protection of skin from UVB-mediated damage. Therefore, it is necessary to further verify the role of HO-1 induction using different wave lengths of UVA, in order to modify HO-1 levels in combination with the specific wave length of UVA1 to enhance the efficiency of treating unpleasant skin conditions. Further, this may provide valuable guidance on skin protection against UV exposure.

\section{Acknowledgements}

We are very grateful to Dr. Rex Tyrrell for stimulating discussion. This work was supported by Scientific Research Foundation for the Returned Overseas Chinese Scholars, State Education Ministry of China (2011-508), National High Technology Research and Development Program of China (Grant No. 2011AA02A103), and National Natural Science Foundation of China (Grant No. 11032012, 30870608).

\section{References}

1. Tyrrell RM. Solar ultraviolet a radiation: An oxidizing skin carcinogen that activates heme oxygenase- 1 . Antioxid Redox Signal. 2004; 6:835-840.

2. Tyrrell RM, Reeve VE. Potential protection of skin by acute UVA irradiation-From cellular to animal models. Prog Biophys Mol Biol. 2006; 92:86-91.

3. Zhong JL, Yang L, Lü F, Xiao H, Xu R, Wang L, Zhu F, Zhang Y. UVA, UVB and UVC induce differential response signaling pathways converged on the eIF2 $\alpha$ phosphorylation. Photochem Photobiol. 2011; 87:1092-1104.

4. Young AR. Acute effects of UVR on human eyes and skin. Prog Biophys Mol Biol. 2006; 92:80-85.

5. Seité S, Fourtanier A, Moyal D, Young AR. Photodamage to human skin by suberythemal exposure to solar ultraviolet radiation can be attenuated by sunscreens: A review. Br J Dermatol. 2010; 163:903-914.

6. Damian DL, Matthews YJ, Halliday GM. Topical riboflavin attenuates ultraviolet B- and ultraviolet A-induced immunosuppression in humans. Photodermatol Photoimmunol Photomed. 2010; 26:66-69.

7. Damian DL, Matthews YJ, Phan TA, Halliday GM. An action spectrum for ultraviolet radiation-induced immunosuppression in humans. Br J Dermatol. 2011; 164:657-659.

8. Moyal DD, Fourtanier AM. Broad-spectrum sunscreens provide better protection from solar ultravioletsimulated radiation and natural sunlight-induced immunosuppression in human beings. J Am Acad Dermatol. 2008; 58 (Suppl 2):S149-S154.

9. Reeve VE, Allanson M, Cho JL, Arun SJ, Domanski D. Interdependence between heme oxygenase-1 induction and estrogen-receptor-beta signaling mediates photoimmune protection by UVA radiation in mice. $\mathrm{J}$ Invest Dermatol. 2009; 129:2702-2710.

10. Ibuki Y, Allanson M, Dixon KM, Reeve VE. Radiation sources providing increased UVA/UVB ratios attenuate the apoptotic effects of the UVB waveband UVA-dosedependently in hairless mouse skin. J Invest Dermatol. 2007; 127:2236-2244.

11. Maines MD. New insights into biliverdin reductase functions: linking heme metabolism to cell signaling. Physiology (Bethesda). 2005; 20:382-389.

12. Gilroy DW, Lawrence T, Perretti M, Rossi AG. Inflammatory resolution: New opportunities for drug discovery. Nat Rev Drug Discov. 2004; 3:401-416.

13. Reeve VE, Tyrrell RM. UVA and inducible protection. In: Biophysical and Physiological Effects of Solar Radiation on Human Skin, Royal Soc for Chem (Giacomoni PU, ed.). RSC Cambridge, UK, 2007; pp. 293-310

14. Reeve VE, Tyrrell RM, Allanson M, Domanski D, Blyth 
L. The role of interleukin-6 in UVA protection against UVB-induced immunosuppression. J Invest Dermatol. 2009; 129:1539-1546.

15. Reeve VE, Allanson M, Domanski D, Painter N. Gender differences in UV-induced inflammation and immunosuppression in mice reveal male unresponsiveness to UVA radiation. Photochem Photobiol Sci. 2011; DOI: 10.1039/C1PP05224A [Epub ahead of print].

16. Matthews YJ, Halliday GM, Phan TA, Damian DL. Wavelength dependency for UVA-induced suppression of recall immunity in humans. J Dermatol Sci. 2010; 59:192-197.

17. Skov L, Villadsen L, Ersbøll BK, Simon JC, Barker JN, Baadsgaard O. Long-wave UVA offers partial protection against UVB-induced immune suppression in human skin. APMIS. 2000; 108:825-830.

18. Nghiem DX, Kazimi N, Clydesdale G, Ananthaswamy HN, Kripke ML, Ullrich SE. Ultraviolet a radiation suppresses an established immune response: Implications for sunscreen design. J Invest Dermatol. 2001; 117:1193-1199.

19. Hojerová J, Medovcíková A, Mikula M. Photoprotective efficacy and photostability of fifteen sunscreen products having the same label SPF subjected to natural sunlight. Int J Pharm. 2011; 408:27-38.

20. Halliday GM, Byrne SN, Kuchel JM, Poon TS, Barnetson RS. The suppression of immunity by ultraviolet radiation: UVA, nitric oxide and DNA damage. Photochem Photobiol Sci. 2004; 3:736-740.

21. Rana S, Rogers LJ, Halliday GM. Immunosuppressive ultraviolet-A radiation inhibits the development of skin memory CD8 T cells. Photochem Photobiol Sci. 2010; 9:25-30.

22. Allanson N, Reeve VE. Photoimmunoprotection by UVA (320-400 $\mathrm{nm}$ ) radiation is determined by UVA dose and is associated with cutaneous cyclic guanosine monophosphate. J Invest Dermatol. 2006; 126:191-197.

23. Tian FF, Zhang FF, Lai XD, Wang LJ, Yang L, Wang X, Singh G, Zhong JL. Nrf2-mediated protection against UVA radiation in human skin keratinocytes. Biosci Trends. 2011; 5:23-29.

24. Zhong JL, Raval C, Edwards GP, Tyrrell RM. A role of Bach1 and HO-2 in suppression of basal and UVAinduced HO-1 expression in human skin keratinocytes. Free Radic Biol Med. 2010; 48:196-206.

25. Soares MP, Marguti I, Cunha A, Larsen R. Immunoregulatory effects of HO-1: How does it work? Curr Opin Pharmacol. 2009; 9:482-489.

26. Keyse SM, Tyrrell RM. Heme oxygenase is the major 32-kDa stress protein induced in human skin fibroblasts by UVA radiation, hydrogen peroxide, and sodium arsenite. Proc Natl Acad Sci U S A. 1989; 86:99-103.

27. Gibbs PE, Maines MD. Biliverdin inhibits activation of NF-kappaB: Reversal of inhibition by human biliverdin reductase. Int J Cancer. 2007; 121:2567-2574.

28. Tyrrell RM. Modulation of gene expression by the oxidative stress generated in human skin cells by UVA radiation and the restoration of redox homeostasis. Photochem Photobiol Sci. 2011; [Epub ahead of print].

29. Balla J, Vercellotti GM, Jeney V, Yachie A, Varga Z, Jacob HS, Eaton JW, Balla G. Heme, heme oxygenase, and ferritin: How the vascular endothelium survives (and dies) in an iron-rich environment. Antioxid Redox Signal. 2007; 9:2119-2137.
30. Aroun A, Zhong JL, Tyrrell RM, Pourzand C. Iron, oxidative stress and the example of solar ultraviolet A radiation. Photochem Photobiol Sci. 2011; [Epub ahead of print].

31. Hirota A, Kawachi Y, Itoh K, Nakamura Y, Xu X, Banno T, Takahashi T, Yamamoto M, Otsuka F. Ultraviolet A irradiation induces NF-E2-related factor 2 activation in dermal fibroblasts: protective role in UVA-induced apoptosis. J Invest Dermatol. 2005; 124:825-832.

32. Shin JW, Ohnishi K, Murakami A, Lee JS, Kundu JK, Na HK, Ohigashi H, Surh YJ. Zerumbone induces heme oxygenase-1 expression in mouse skin and cultured murine epidermal cells through activation of Nrf2. Cancer Pre Res (Phila). 2011; 4:860-870.

33. Zhong JL, Edwards GP, Raval C, Li HB and Tyrrell RM. The role of Nrf2 in Ultraviolet A mediated heme oxygenase 1 induction in human skin fibroblasts. Photochem Photobiol Sci. 2010; 9:18-24.

34. Listopad J, Asadullah K, Sievers C, Ritter T, Meisel C, Sabat R, Döcke WD. Heme oxygenase-1 inhibits T cell-dependent skin inflammation and differentiation and function of antigen-presenting cells. Exp Dermatol. 2007; 16:661-670.

35. Chora AA, Fontoura P, Cunha A, Pais TF, Cardoso S, Ho PP, Lee LY, Sobel RA, Steinman L, Soares MP. Heme oxygenase-1 and carbon monoxide suppress autoimmune neuroinflammation. J Clin Invest. 2007; 117:438-447.

36. Shimizu K, Bae SJ, Hara T, Iwata Y, Yamaoka T, Komura K, Muroi E, Takenaka M, Ogawa F, Sato S. Involvement of gaseous low molecular monoxides in the cutaneous reverse passive Arthus reaction: Cytoprotective action of carbon monoxide. Clin Exp Immunol. 2008; 153:245-257.

37. Reeve VE, Bosnic M, Boehm-Wilcox C, Nishimura N, Ley RD. Ultraviolet A radiation (320-400 nm) protects hairless mice from immunosuppression induced by ultraviolet B radiation $(280-320 \mathrm{~nm})$ or cis-urocanic acid. Int Arch Allergy Immunol. 1998; 115:316-322.

38. Reeve VE, Tyrrell RM. Heme oxygenase induction mediates the photoimmunoprotective activity of UVA radiation in the mouse. Proc Natl Acad Sci U S A. 1999; 96:9317-9321.

39. Allanson M, Reeve VE. Immunoprotective UVA (320-400 nm) irradiation upregulates heme oxygenase-1 in the dermis and epidermis of hairless mouse skin. J Invest Dermatol. 2004; 122:1030-1036.

40. Reeve VE, Domanski D, Slater M. Radiation sources providing increased UVA/UVB ratios induce photoprotection dependent on the UVA dose in hairless mice. Photochem Photobiol. 2006; 82:406-411.

41. Reeve VE, Domanski D. Refractoriness of UVA-induced protection from photoimmunosuppression correlates with heme oxygenase response to repeated UVA exposure. Photochem Photobiol. 2002; 76:401-405.

42. Noël A, Tyrrell RM. Development of refractoriness of induced human heme oxygenase-1 gene expression to reinduction by UVA irradiation and hemin. Photochem Photobiol. 1997; 66:456-463.

43. Reeve VE, Domanski D. Immunoprotective haem oxygenase induction by ultraviolet A (320-400 nm) radiation in the mouse is inhibited in interferon-gamma null mice. Br J Dermatol. 2003; 148:1189-1193.

44. Allanson M, Reeve VE. Ultraviolet A (320-400 nm) modulation of ultraviolet B (290-320 nm)-induced immune suppression is mediated by carbon monoxide. $\mathrm{J}$ 
Invest Dermatol. 2005; 124:644-650.

45. Wojas-Pelc A, Marcinkiewicz J. What is a role of haeme oxygenase- 1 in psoriasis? Current concepts of pathogenesis. Int J Exp Pathol. 2007; 88:95-102.

46. York NR, Jacobe HT. UVA1 phototherapy: A review of mechanism and therapeutic application. Int J Dermatol. 2010; 49:623-630.

47. Pereira N, Santiago F, Oliveira H, Figueiredo A. Lowdose UVA(1) phototherapy for scleroderma: What benefit can we expect? J Eur Acad Dermatol Venereol. 2011; DOI: 10.1111/j.1468-3083.2011.04137.x. [Epub ahead of print].
48. Szegedi A, Simics E, Aleksza M, Horkay I, Gaál K, Sipka S, Hunyadi J, Kiss E. Ultraviolet-A1 phototherapy modulates $\mathrm{Th} 1 / \mathrm{Th} 2$ and $\mathrm{Tc} 1 / \mathrm{Tc} 2$ balance in patients with systemic lupus erythematosus. Rheumatology (Oxford). 2005; 44:925-931.

49. Su O, Onsun N, Onay HK, Erdemoglu Y, Ozkaya DB, Cebeci F, Somay A. Effectiveness of medium-dose ultraviolet A1 phototherapy in localized scleroderma. Int J Dermatol. 2011; 50:1006-1013.

(Received July 14, 2011; Revised November 7, 2011;Rerevised November 30, 2011; Accepted December 7, 2011) 